\title{
The Related Properties of Generalized Orthogonal Group in Specific Normed Linear Spaces
}

\author{
Siyuan Wu \\ Department of Mathematics, Harbin University of Science and Technology, Harbin, China \\ Email: 2330475414@qq.com
}

How to cite this paper: Wu, S.Y. (2022) The Related Properties of Generalized Orthogonal Group in Specific Normed Linear Spaces. Journal of Applied Mathematics and Physics, 10, 1-10.

https://doi.org/10.4236/jamp.2022.101001

Received: November 29, 2021

Accepted: January 4, 2022

Published: January 7, 2022

Copyright $\odot 2022$ by author(s) and Scientific Research Publishing Inc. This work is licensed under the Creative Commons Attribution International License (CC BY 4.0).

http://creativecommons.org/licenses/by/4.0/

\section{(c) (i) Open Access}

\begin{abstract}
Firstly, in the general normed linear space, the concepts of generalized isosceles orthogonal group, generalized Birkhoff orthogonal group, generalized Roberts orthogonal group, strong Birkhoff orthogonal group and generalized orthogonal basis are introduced. Secondly, the conclusion that any two nonzero generalized orthogonal groups must be linearly independent group is proven. And the existence of nonzero generalized orthogonal group and its linear correlation are discussed preliminarily, as well as some related properties of nonempty generalized orthogonal group in specific normed linear space namely the $l_{p}$ space.
\end{abstract}

\section{Keywords}

Normed Linear Space, Generalized Orthogonal Group, Generalized Birkhoff Orthogonal Group, Generalized Isosceles Orthogonal Group, Generalized Roberts Orthogonal Group

\section{Related Properties of Generalized Orthogonal Group and Generalized Orthogonal Basis}

Orthogonality is an important study content of inner product space. On the one hand, as scholars have deepened their understanding of functional analysis, especially the understanding of Banach geometric theory, generalized orthogonal theory in the normed linear space was established and corresponding studies were carried out. The essential characteristics of orthogonality were applied to extend orthogonality from inner product space to general normed linear space. On the other hand, orthogonal groups, orthogonal bases and orthogonalization of vector groups have been extensively studied, making the whole system more 
complete. As a result, the study achievements within these fields have played an important role in the improvement of the inner product space theory. Naturally, scholars are making efforts to extend related theories to general normed linear spaces. At the same time, the application of related orthogonality of nonorthogonal function expansion is also rising [1].

In this paper, based on the research achievements of orthogonal theory, the concepts of generalized orthogonal group and generalized orthogonal basis in the general normed linear space were introduced. The conclusion that any two nonzero generalized orthogonal groups must be linearly independent groups was proven. An example showing that there exist four nonzero Birkhoff orthogonal groups in two-dimensional space was given. Also, this example further drew our attention to the study of generalized orthogonal groups' properties. Next, the existence of nonzero generalized orthogonal groups and its linear correlation were discussed, as well as some related questions of nonzero generalized orthogonal groups in $l_{p}$ space.

The research on orthogonal and isometric mapping has attracted much attention as early as the beginning of the 20th century. In 1934, B. D. Roberts proposed the concept of Roberts orthogonality [2]. The specific definition is as follows.

Definition 1.1 ([2]) Let $X$ be a normed linear space, $x, y \in X$, if the equation

$$
\|x+\alpha y\|=\|x-\alpha y\|
$$

holds for any real number $\alpha$. Then $x$ is Roberts orthogonal to $y$, denoted by $x \perp_{R} y$.

Definition 1.2 ([3]) Let $X$ be a normed linear space, $x, y \in X$, if they satisfy

$$
\|x+y\|=\|x-y\| \text {. }
$$

Then $x$ is isosceles orthogonal or James orthogonal to $y$, denoted by $x \perp_{I} y$.

According to the property of "The perpendicular segment between the point and the line is shortest", the concept of Birkhoff orthogonality in normed linear space was proposed by G. Birkhoff in 1935.

Definition 1.3 ([4]) Let $X$ be a normed linear space, $x, y \in X$, if the inequelity

$$
\|x+\alpha y\| \geq\|x\|
$$

holds for any $\alpha$ in the range of real number. Then $x$ is Birkhoff orthogonal to $y$, denoted by $x \perp_{B} y$.

Because R. C. James has made outstanding contributions to the study of Birkhoff Orthogonal, Birkhoff Orthogonal is also known as James Orthogonal or Birkhoff-James Orthogonal.

Definition 1.4 ([5]) Suppose $M$ and $N$ are two subspaces of $X$, if for any $x \in M$ and $y \in N$, there exist $x \perp_{B} y$, then $M$ is Birkhoff orthogonal to $N$, denoted by $M \perp_{B} N .\langle\{x\}\rangle \perp_{B} N$ and $M \perp_{B}\langle\{y\}\rangle$ are abbreviated as $x \perp_{B} N$ and $M \perp_{B} y$ in particular. 
Definition 1.5 ([5]) A norm on $\mathbb{R}^{n}$ is standard when satisfies

$$
\|\cdot\|_{1} \leq\|\cdot\| \leq\|\cdot\|_{\infty} \text {. }
$$

Definition 1.6 ([6]) Let $X$ be a linear space and $\|\cdot\|: X \mapsto \mathbb{R}$ be a functional on $X$. If for any $x, y \in X$ and $\alpha \in \mathbb{R},\|\cdot\|$ satisfies the following conditions:

1) $\|x\| \geq 0,\|x\|=0 \Leftrightarrow x=0$;

2) $\|x+y\| \leq\|x\|+\|y\|$ (Triangle inequality);

3) $\|\alpha x\| \leq|\alpha|\|x\|$ (Absolute homogeneity).

Then $\|\cdot\|$ is called a norm on $X$, and $(X,\|\cdot\|)$ is called a normed linear space. When the norm is not emphasized, $X$ is used to represent $(X,\|\cdot\|)$. A real finite-dimensional normed linear space is called a Minkowski space, and a two-dimensional Minkowski space is called a Minkowski plane.

Based on the definition and related theorems of orthogonality, as scholars keep studying in the field of orthogonal elements, conclusions in specific Orlicz sequence space were correspondingly drawn [7] [8]. Along with the further understanding of orthogonal elements, the concept of orthogonal groups was proposed for the reason that orthogonal elements are not commutative. In the inner product space, the generalized orthogonal theory was relatively complete. And in algebra, studies on vector groups were already carried out. Naturally, the generalized orthogonal groups whose properties are derived from orthogonality while keep their own differences were studied in this paper. Considering of different orthogonal properties, different orthogonal groups were correspondingly proposed. And the related properties of orthogonal groups were studied in specific normed linear space.

Based on these definitions, in the general normed linear space, the concepts of generalized isosceles orthogonal group, generalized Birkhoff orthogonal group, generalized Roberts orthogonal group, strong Birkhoff orthogonal group and generalized orthogonal basis are introduced correspondingly as follows:

Definition 1.7 Let $X$ be a normed linear space, $A=\left(\alpha_{1}, \alpha_{2}, \cdots, \alpha_{m}\right)$ is a vector group of $X$, if there exist $\alpha_{i} \perp_{B} \alpha_{j}$ or $\alpha_{j} \perp_{B} \alpha_{i}$ for any $\alpha_{i}, \alpha_{j} \in A$, then the vector group $A$ is a generalized Birkhoff orthogonal group of $X$.

Definition 1.8 Let $X$ be a normed linear space, $A=\left(\alpha_{1}, \alpha_{2}, \cdots, \alpha_{m}\right)$ is a vector group of $X$, if there exist $\alpha_{i} \perp_{B} \alpha_{j}$ and $\alpha_{j} \perp_{B} \alpha_{i}$ for any $\alpha_{i}, \alpha_{j} \in A$, then the vector group $A$ is a strong Birkhoff or commutative Birkhoff orthogonal group of $X$.

Definition 1.9 Let $X$ be a normed linear space, $A=\left(\alpha_{1}, \alpha_{2}, \cdots, \alpha_{m}\right)$ is a vector group of $X$, if there exist $\alpha_{i} \perp_{I} \alpha_{j}$ for any $\alpha_{i}, \alpha_{j} \in A$. Moreover, considering the symmetry of isosceles orthogonal, then the vector group $A$ is a strong Birkhoff or commutative Birkhoff orthogonal group of $X$.

Definition 1.10 Let $X$ be a normed linear space, $A=\left(\alpha_{1}, \alpha_{2}, \cdots, \alpha_{m}\right)$ is a vector group of $X$, if there exist $\alpha_{i} \perp_{R} \alpha_{j}$ or $\alpha_{j} \perp_{R} \alpha_{i}$ for any $\alpha_{i}, \alpha_{j} \in A$, then the vector group $A$ is a generalized Roberts orthogonal group of $X$.

Definition 1.11 Let $A$ be a generalized orthogonal group on normed linear space $X$. If $A$ is also a set of basis on $X$, then $A$ is a generalized orthogonal basis 
of $X$.

Theorem 1.1 Any two elements in any nonzero generalized orthogonal group in a normed linear space are linearly independent.

Proof In the following, we distinguish four cases.

Case 1: If $(x, y)$ is a nonzero Birkhoff orthogonal group. Assuming that $(x, y)$ is linearly related, then there exist $y=k x$,

$$
\|x+\alpha y\|=\|x+\alpha k x\|=\|(1+\alpha k) x\| .
$$

This is a contradiction to the definition of Birkhoff orthogonal. Thus, if $(x, y)$ is a nonzero Birkhoff orthogonal group, then it is linearly independent.

Case 2: If $(x, y)$ is a nonzero strong Birkhoff orthogonal group. Assuming that $(x, y)$ is linearly related, then there exist $y=k x$,

$$
\|x+\alpha y\|=\|(1+\alpha k) x\| \text { and }\|y+\alpha x\|=\|(k+\alpha) x\| .
$$

This is a contradiction to the definition of Birkhoff orthogonal. Thus, if $(x, y)$ is a nonzero strong Birkhoff orthogonal group, then it is linearly independent.

Case 3: If $(x, y)$ is a nonzero isosceles orthogonal group. Similarly, assuming that $(x, y)$ is linearly related, then there exist

$$
\|x+y\|=\|(1+k) x\| \text { and }\|x-y\|=\|(1-k) x\| .
$$

This is a contradiction to the definition of isosceles orthogonal. Thus, if $(x, y)$ is a nonzero isosceles orthogonal group, then it is linearly independent.

Case 4: If $(x, y)$ is a nonzero Roberts orthogonal group. Assuming that $(x, y)$ is linearly related, then there exist

$$
\|x+\alpha y\|=\|(1+\alpha k) x\| \text { and }\|x-\alpha y\|=\|(1-\alpha k) x\| .
$$

This is a contradiction to the definition of Roberts orthogonal. Thus, if $(x, y)$ is a nonzero Roberts orthogonal group, then it is linearly independent.

The generalized orthogonal group of two elements is linearly independent, so it is natural for us to consider whether there are linear independent orthogonal group of three or more elements in two-dimensional space.

Reasoning 1.1 Any generalized orthogonal group containing two nonzero elements in a two-dimensional space is a generalized orthogonal basis.

However, it should be noted that in a three-dimensional space, a generalized orthogonal group containing three elements is not necessarily a generalized orthogonal basis. Specific examples are given below.

Theorem 1.2 Let $X$ be the inner product space and $\operatorname{dim} X=n, x_{1}, x_{2}, \cdots, x_{n+1}$ are orthogonal to each other, then in there exists at least one zero element.

Proof Proof by contradiction assuming that $x_{1}, x_{2}, \cdots, x_{n+1}$ are nonzero and orthogonal to each other. Since $\operatorname{dim} X=n$, we have

$$
a_{1} x_{1}+a_{2} x_{2}+\cdots+a_{n+1} x_{n+1}=0 \text {. }
$$

Thus,

$$
\left\langle 0 \cdot x_{1}\right\rangle=\left\langle a_{1} x_{1} \cdot x_{1}\right\rangle+\left\langle a_{2} x_{2} \cdot x_{1}\right\rangle+\cdots+\left\langle a_{n+1} x_{n+1} \cdot x_{1}\right\rangle=a_{1}\left\|x_{1}\right\|^{2} .
$$


Since $\left\|x_{1}\right\|>0$, we have $a_{1}=0$.

Similarly, we have $a_{2}=0, \cdots, a_{n+1}=0$. This contradicts the linear correlation of $x_{1}, x_{2}, \cdots, x_{n+1}$, hence the theorem is proved.

Theorem 1.3 ([9]) For any convex quadrilateral $a b c d$ on the Minkowski plane $X$, the sum of its diagonals is no less than the sum of lengths of any set of opposite sides, that is

$$
\begin{aligned}
& \|c-a\|+\|d-b\| \geq\|b-a\|+\|d-c\| \\
& \|c-a\|+\|d-b\| \geq\|c-b\|+\|d-a\|
\end{aligned}
$$

Formula (1-1) holds if and only if

$$
[(c-a) /\|c-a\|,(b-d) /\|b-d\|] \subset S_{X} .
$$

Theorem 1.4 ([2]) Let $C$ be a convex body in $\mathbb{R}^{n}(n \geq 3)$. The following propositions are equivalent

1) $C$ is an ellipsoid;

2) For any straight line $L$ passing through the origin, there is a hyperplane $H$ such that $C+L=C \cap H+L$.

Theorem 1.5 Let $X$ be a two-dimensional real normed linear space. If there exists a generalized Roberts orthogonal group on $X$, then $\|x\|\|y\|\|z\|=0$ holds.

Theorem 1.6 ([10]) Let $E$ be a two-dimensional real normed linear space. If there exist $x, y, z \in E, \quad x \perp_{I} y, x \perp_{I} z$ and $y \perp_{I} z$, then $\|x\|\|y\|\|z\|=0$.

Note that the theorem above does not hold for Birkhoff orthogonality.

Example 1.1 ([11]) In $\mathbb{R}^{2}$ with the norm $\left\|\left(x_{1}, x_{2}\right)\right\|=\max \left\{\left|x_{1}\right|,\left|x_{2}\right|\right\}$, vector $x=(1,-1), \quad y=(1,1)$ and $z=(0,1)$ satisfy $x \perp_{B} y, x \perp_{B} z$ and $y \perp_{B} z$.

Example 1.2 Let $X$ be a linear space formed by a continuous function $f(\alpha)=\gamma \alpha+\eta \alpha^{2}$ on a closed interval $[0,1]$, and specify

$$
\|f\|=\max _{\alpha \in[0,1]}\left|\gamma \alpha+\eta \alpha^{2}\right| \text {. }
$$

Then $(X,\|\cdot\|)$ is a two-dimensional normed linear space; the two vectors $X$ and $y$ in $X$ are Roberts orthogonal to each other if and only if $\|x\| \cdot\|y\|=0$.

Example 1.3 Let $X=\left(\mathbb{R}^{2},\|\cdot\|\right)$, if the norm $\left\|\left(x_{1}, x_{2}\right)\right\|=\max \left\{\left|x_{1}\right|,\left|x_{2}\right|\right\}$ satisfies $x=(\alpha,-\alpha), \quad y=(\alpha, \alpha), \quad z=(0, u)$ and $w=(v, 0)$, where $\alpha, u, v \in \mathbb{R}$, then $x \perp_{B} y, \quad x \perp_{B} z, \quad x \perp_{B} w, y \perp_{B} w, y \perp_{B} z, \quad z \perp_{B} w$ and $\|x\|\|y\|\|z\|\|w\| \neq 0$.

Example 1.3 shows that the number of elements in a nonzero generalized orthogonal group can be greater than the number of elements in the basis.

\section{Generalized Orthogonal Group in $l_{p}$ Space}

In this part, the existence of nonzero generalized orthogonal groups and its linear correlations were applied in discussing the related questions in the specific normed linear space namely the $l_{p}$ space. The existences of generalized orthogonal elements and generalized orthogonal groups in $l_{p}^{2}$ and $l_{p}^{3}$ space were proven. 
Theorem 2.1 Let $X=\left(\mathbb{R}^{2},\|\cdot\|_{p}\right)$, then any linearly independent group on $X$ can be Schmidt orthogonalized.

Proof Let $X=\left(\mathbb{R}^{2},\|\cdot\|_{p}\right)$ and $J$ is the standard dual mapping on $X$. Select $\tilde{J}(z) \in J(z)$ which is a standard dual mapping satisfying $\tilde{J}(\lambda z)=\lambda \tilde{J}(z)$ for $\forall z \in S_{X}$ and $\forall \lambda \in \mathbb{R}$.

It follows that $\tilde{J}$ is one option of standard dual mapping.

$\forall x, y \in X$, let $[x \mid y]=\tilde{J}(y)(x)$, and we have $[x \mid x]=\tilde{J}(x)(x)=\|x\|^{2}$. Thus, $[\cdot \mid \cdot]$ is a semi-inner product of a generation norm on $X$.

Assume $u, v \in S_{X}$, and we have

$$
w=v-u \cdot[v \mid u] /[u \mid u] .
$$

Thus,

$$
[w \mid u]=\tilde{J}(u)(w)=\tilde{J}(u)\left\{v-(u \cdot \tilde{J}(u)(v)) /\|u\|^{2}\right\}=\tilde{J}(u)(v)-\tilde{J}(u)(v)=0 .
$$

We have $u \perp_{B} w$ which implies that $u, w$ is a set of orthogonal basis.

Proposition 2.1 ([2]) If $X$ is a Minkowski plane, then for any vector $X$ in $X$, there exists a corresponding $H$ in $X$ ( $H$ is a hyperplane passing through the origin) so that any vector in $H$ is Birkhoff orthogonal to $x$ (denoted by $H \perp_{B} X$ ).

However, when the dimension of the space is greater than two, the situation is completely different.

Lemma 2.1 ([2]) Let $X$ be a normed linear space with dimensions great than or equal to three. If $H$ exists for any vector $x$ such that $H \perp_{B} X \quad(H$ is a hyperplane through the origin), then $x$ is an inner product space.

Next, we were trying to find the generalized orthogonal element in $l_{p}^{2}$ space using the same proof methods as Lemma 2.1.

Proposition 2.2 Birkhoff orthogonal element of $l_{p}^{2}$ space.

$\forall x \in l_{p}^{2}, \exists f \in X^{k} /\{0\}$, satisfying $f(x)=\|x\|$ and

$$
X^{k}=(\|x\| \cdot f) /\|f\| \text {. }
$$

Then we have $X^{k}(x)=\cdots=\|x\|^{2}$.

Let $y \in l_{p}^{2}$ satisfying $X^{k}(y)=0$, and it follows that

$$
\|x+\alpha y\| \geq X^{k}(x+\alpha y) /\left\|x^{k}\right\|=x^{k}(x) /\left\|x^{k}\right\|=\|x\| .
$$

Hence $x \perp_{B} y$.

In the two-dimensional subspace $X_{\phi}$ of $l_{p}$ space, let $x=\left(x_{1}, x_{2}, 0, \cdots, 0\right)$, $y=\left(y_{1}, y_{2}, 0, \cdots, 0\right)$ and $\left|x_{1}\right|^{p}+\left|x_{2}\right|^{p}=1$, which is $x \in S_{X}$.

Let $\left|x_{1}\right|^{p}=u$ and $\left|x_{2}\right|^{p}=v$, we have

$$
\begin{gathered}
\left|x_{1}\right|=u^{1 / p} \text { and }\left|x_{2}\right|=v^{1 / p}, \\
\|x+\alpha y\|=\left(\left|x_{1}+\alpha y_{1}\right|^{p}+\left|x_{2}+\alpha y_{2}\right|^{p}\right)^{1 / p}, \\
\|x\|=\left(\left|x_{1}\right|^{p}+\left|x_{2}\right|^{p}\right)^{1 / p},
\end{gathered}
$$




$$
\mathrm{d} x_{1} / \mathrm{d} x_{2}=\left(\mathrm{d} x_{1} / \mathrm{d} u\right) \cdot(\mathrm{d} u / \mathrm{d} v) \cdot\left(\mathrm{d} v / \mathrm{d} x_{2}\right)=-\left|x_{1}\right|^{p-1} /\left|x_{2}\right|^{p-1} .
$$

If $\|x+\alpha y\| \geq\|x\|$ holds, then

$$
\left(\left|x_{1}+\alpha y_{1}\right|^{p}+\left|x_{2}+\alpha y_{2}\right|^{p}\right)^{1 / p} \geq\left(\left|x_{1}\right|^{p}+\left|x_{2}\right|^{p}\right)^{1 / p}
$$

must hold for any $\alpha$.

Since $y=k x+b$, we have that

$$
\left(1,-\left|x_{2}\right|^{p-1} /\left|x_{1}\right|^{p-1}\right)
$$

which is an orthogonal point pair. Hence

$$
\begin{gathered}
x= \pm(0,1), \quad y= \pm(1,0) \\
x= \pm(1 / \sqrt[p]{2}, 1 / \sqrt[p]{2}), \quad y= \pm(-1 / \sqrt[p]{2}, 1 / \sqrt[p]{2}) .
\end{gathered}
$$

Proposition 2.3 Roberts orthogonal element of $l_{p}^{2}$ space.

Let $x=(a, b)$ be a point on the unit sphere of two-dimensional $l_{p}$ space and $x \in S_{X}$, it follows that

$$
|a|^{p}+|b|^{p}=1
$$

Let $b=b(a)$, and we have

$$
\begin{gathered}
p|a|^{p-1}+b^{\prime}(a) p(|b|)^{p-1}=0, \\
b^{\prime}(a)=-p|a|^{p-1} / p(|b|)^{p-1}=-|a|^{p-1} /|b|^{p-1} .
\end{gathered}
$$

which implies that the hyperplane $H=\left\{x-\lambda|a|^{p-1} /|b|^{p-1}, \lambda \in R\right\}$ supports the ball at $x$.

When $1<p<\infty$, the unit sphere is strictly convex, then $x$ is Birkhoff orthogonal to $y$ for any $X$. If $x \perp_{B} y$, then $y \in H-x$ (the point $y$ in the hyperplane except for $x$ ).

Let $x \perp_{R} y$, and we have

$$
\|x+\lambda y\|=\|x-\lambda y\| \text { and } y=\left(1,-|a|^{p-1} /|b|^{p-1}\right) .
$$

Thus,

$$
|a+\lambda|^{p}+\left.\left.|b-\lambda| a\right|^{p-1} \cdot|b|^{p-1}\right|^{p}=|a-\lambda|^{p}+\left.\left.|b+\lambda| a\right|^{p-1} \cdot|b|^{p-1}\right|^{p} .
$$

When $|a|=|b|$ or $a b>0$, there exists elements Roberts orthogonal to $x$, which are

$$
\begin{gathered}
x=(0,1), \quad y=(1,0) ; \quad x=(1,0), \quad y=(0,1) ; \\
x=(0,-1), \quad y=(-1,0) ; \quad x=(-1,0), \quad y=(0,-1) ; \\
x=\left(1 / 2^{p}, 1 / 2^{p}\right), \quad y=(1,-1) ; \quad x=\left(1 / 2^{p},-1 / 2^{p}\right), \quad y=(1,1) ; \\
x=\left(-1 / 2^{p}, 1 / 2^{p}\right), \quad y=(-1,-1) ; \quad x=\left(-1 / 2^{p},-1 / 2^{p}\right), \quad y=(-1,1) .
\end{gathered}
$$

Proposition 2.4 If $x=(\alpha, \beta)$ and $y= \pm(-\beta, \alpha) \in l_{p}^{2}(1<p<\infty)$, then $x \perp_{I} y$. 
Proof In the following, we distinguish two cases.

Case 1: Since $x=(\alpha, \beta)$ and $y=(-\beta, \alpha)$, we have

$$
x+y=(\alpha-\beta, \beta+\alpha) \text { and } x-y=(\alpha+\beta, \beta-\alpha) \text {. }
$$

It follows that

$$
\|x+y\|=\sqrt[p]{|\alpha-\beta|^{p}+|\beta+\alpha|^{p}} \text { and }\|x-y\|=\sqrt[p]{|\alpha+\beta|^{p}+|\beta-\alpha|^{p}} \text {. }
$$

Case 2: Since $x=(\alpha, \beta)$ and $y=(\beta,-\alpha) \in l_{p}^{2}(1<p<\infty)$, we have

$$
x+y=(\alpha+\beta, \beta-\alpha) \text { and } x-y=(\alpha-\beta, \beta+\alpha) \text {. }
$$

It follows that

$$
\|x+y\|=\sqrt[p]{|\alpha+\beta|^{p}+|\beta-\alpha|^{p}} \text { and }\|x-y\|=\sqrt[p]{|\alpha-\beta|^{p}+|\beta+\alpha|^{p}} .
$$

Proposition 2.5 The isosceles orthogonality on space $l_{p}^{3}(1<p<\infty)$ can be obtained from the foregoing.

Proof In the following, we distinguish three cases.

Case 1: If $x=(\alpha, \beta, \gamma)$ and $y= \pm(-\beta, \alpha, 0) \in l_{p}^{3}$, then $x \perp_{I} y$.

Case 2: If $x=(\alpha, \beta, \gamma)$ and $y= \pm(0,-\gamma, \beta)$, then $x \perp_{I} y$.

1) If $x+y=(\alpha, \beta-\gamma, \gamma+\beta)$ and $x-y=(\alpha, \beta+\gamma, \gamma-\beta)$, then we have $\|x+y\|=\sqrt[p]{|\alpha|^{p}+|\beta-\gamma|^{p}+|\gamma+\beta|^{p}}$ and $\|x-y\|=\sqrt[p]{|\alpha|^{p}+|\beta+\gamma|^{p}+|\gamma-\beta|^{p}}$.

Hence $\|x+y\|=\|x-y\|$.

2) If $x+y=(\alpha, \beta+\gamma, \gamma-\beta)$ and $x-y=(\alpha, \beta-\gamma, \gamma+\beta)$, similarly,

$$
\|x+y\|=\|x-y\| \text {. }
$$

Case 3: If $x=(\alpha, \beta, \gamma)$ and $y= \pm(-\gamma, 0, \alpha)$, then $x \perp_{I} y$.

1) If $x+y=(\alpha-\gamma, \beta, \gamma+\alpha)$ and $x-y=(\alpha+\gamma, \beta, \gamma-\alpha)$, then we have

$$
\|x+y\|=\sqrt[p]{|\alpha-\gamma|^{p}+|\beta|^{p}+|\gamma+\alpha|^{p}} \text { and }\|x-y\|=\sqrt[p]{|\alpha+\gamma|^{p}+|\beta|^{p}+|\gamma-\alpha|^{p}} \text {. }
$$

Hence $\|x+y\|=\|x-y\|$.

2) If $x+y=(\alpha+\gamma, \beta, \gamma-\alpha)$ and $x-y=(\alpha-\gamma, \beta, \gamma+\alpha)$, similarly, we have

$$
\|x+y\|=\|x-y\|
$$

Theorem 2.2 There exist at least $n$ elements in $n$-dimensional $l_{p}$ space that are orthogonal to each other and linearly independent.

Example 2.1 Let $X$ be a $l_{p}^{n}$ space, then $A=\left(e_{1}, \cdots, e_{n}\right)$ is the generalized Birkhoff orthogonal group of $X$, is also the generalized isosceles orthogonal group and the generalized Roberts orthogonal group of $X$, and is linearly independent.

Then we naturally consider the existence of generalized orthogonal groups in $l_{p}$ subspaces.

Reasoning 2.1 Let $X$ be a two-dimensional $l_{p}^{2}$ subspace, $A=\left(x_{1}, x_{2}\right)$, $B=\left(y_{1}, y_{2}\right), \quad x_{1}= \pm(0,1), \quad x_{2}= \pm(1,0), \quad y_{1}= \pm(1 / \sqrt[p]{2}, 1 / \sqrt[p]{2})$ and 
$y_{2}= \pm(1 / \sqrt[p]{2}, 1 / \sqrt[p]{2})$. It is proved that $A$ and $B$ are generalized orthogonal groups of $X$, and $x_{1}, x_{2}, y_{1}, y_{2}$ are mutually commutative. Hence $A$ and $B$ can also be called as generalized commutative Birkhoff orthogonal groups of $X$.

Reasoning 2.2 Let $X$ be a two-dimensional $l_{p}^{2}$ subspace, $A=\left(x_{1}, x_{2}\right)$, $B=\left(y_{1}, y_{2}\right), \quad C=\left(z_{1}, z_{2}\right), \quad x_{1}= \pm(0,1), \quad x_{2}= \pm(1,0), \quad y_{1}= \pm\left(1 / 2^{p}, 1 / 2^{p}\right)$, $y_{2}= \pm(1,-1), \quad z_{1}= \pm\left(1 / 2^{p},-1 / 2^{p}\right), \quad z_{2}= \pm(1,1)$. It is proved that $A, B$ and $C$ are generalized Roberts orthogonal groups of $X$, and $x_{1}, x_{2}, y_{1}, y_{2}, z_{1}, z_{2}$ are mutually commutative. Hence $A, B$ and $C$ are called generalized commutative Roberts orthogonal groups of $X$.

Reasoning 2.3 Let $X$ be a two-dimensional $l_{p}^{2}$ subspace, $A=\left(x_{1}, x_{2}\right)$, $x_{1}=(\alpha, \beta)$ and $x_{2}= \pm(-\beta, \alpha)$. It is proved that $A$ is a generalized isosceles orthogonal group of $X$.

Reasoning 2.4 Let $X$ be a $l_{p}^{3}$ space, $A=\left(x_{1}, x_{2}\right), B=\left(y_{1}, y_{2}\right), C=\left(z_{1}, z_{2}\right)$, $x_{1}=(\alpha, \beta, \gamma), \quad x_{2}= \pm(-\beta, \alpha, 0), \quad y_{1}=(\alpha, \beta, \gamma), \quad y_{2}= \pm(0,-\gamma, \beta)$, $z_{1}=(\alpha, \beta, \gamma)$ and $z_{2}= \pm(-\gamma, 0, \alpha)$. It is proved that $A, B$ and $C$ are generalized isosceles orthogonal groups of $X$.

Reasoning 2.5 Let $X$ be a $l_{\infty}^{2}$ space, $A=\left(x_{1}, x_{2}, x_{3}\right), \quad x_{1}=(0,1), \quad x_{2}=(1,0)$ and $x_{3}=(1,1)$. It is proved that $A$ is a generalized Birkhoff orthogonal group of $X$. However, $x_{1}, x_{2}, x_{3}$ are not mutually Brikhoff orthogonal, then $A$ cannot be called as a generalized commutative Birkhoff orthogonal group of $X$.

However, there still remains further study and discussion on the existence of generalized orthogonal groups in other normed linear spaces.

\section{Conclusion}

Based on the concept of orthogonal group in inner product space and some related properties, the definition of generalized orthogonal group in general normed linear space is introduced in this paper. Furthermore, the existence and linear correlation of nonzero generalized orthogonal groups are discussed. Some related problems of nonzero generalized orthogonal groups in specific normed linear space namely the $l_{p}$ space are discussed, and corresponding conclusions are drawn. Also, generalized orthogonal basis in the three-dimensional Orlicz sequence space is discussed, and the isosceles orthogonal basis is extended especially.

\section{Conflicts of Interest}

The author declares no conflicts of interest regarding the publication of this paper.

\section{References}

[1] Szlavik, R., Paquin, D. and Turner III, G. (2017) A Perturbative-Based Generalized Series Expansion in Terms of Non-Orthogonal Component Functions. Applied Mathematics, 8, 106-116. https://doi.org/10.4236/am.2017.81009

[2] Roberts, B.D. (1934) On the Geometry of Abstract Vector Spaces. Tohoku Mathe- 
matic Journal, 39, 42-59.

[3] James, R.C. (1945) Orthogonality in Normed Linear Spaces. Duke Math, 12, 291-301. https://doi.org/10.1215/S0012-7094-45-01223-3

[4] Birkhoff, G. (1935) Orthogonality in Linear Metric Spaces. Duke Math, 1, 169-172. https://doi.org/10.1215/S0012-7094-35-00115-6

[5] Ding, G.G. (2008) Introduction to Banach space. 2nd Edition, China Science Publishing \& Media Ltd., Beijing.

[6] Naszódi, M., Prokaj, V. and Swanepoel, K. (2020) Angular Measures and Birkhoff Orthogonality in Minkowski Planes. Aequationes Mathematicae, 94, 969-977. https://doi.org/10.1007/s00010-020-00715-4

[7] Willian, H.G.C. (2020) Complex Interpolation of Families of Orlicz Sequence Spaces. Israel Journal of Mathematics, 12, 1-22.

[8] Bakery, A.A. and Elmatty, A.R.A. (2020) Some Properties of Pre-Quasi Norm on Orlicz Sequence Space. Journal of Inequalities and Applications, 1, 17-36. https://doi.org/10.1186/s13660-020-02318-8

[9] Webster, R. (1994) Convexity. 1st Edition, Oxford University Press, New York.

[10] Rassias, T.M. (1997) Inner Product Spaces and Applications. Taylor \& Francis Group, United Kingdom.

[11] Alonso, J., Martini, H. and Wu, S.L. (2012) On Birkhoff Orthogonality and Isosceles Orthogonality in Normed Linear Spaces. Aequationes Mathematicae, 83, 153-189. https://doi.org/10.1007/s00010-011-0092-z 